\title{
Association among biofilm formation, virulence gene expression, and antibiotic resistance in Proteus mirabilis isolates from diarrhetic animals in Northeast China
}

\author{
Yadong Sun ${ }^{1,2}$, Shanshan Wen ${ }^{1}$, Lili Zhao ${ }^{3}$, Qiqi Xia ${ }^{1}$, Yue Pan ${ }^{1}$, Hanghang Liu' ${ }^{1}$ Chengwei Wei ${ }^{1}$, Hongyan Chen ${ }^{3}$,
} Junwei $\mathrm{Ge}^{1,4}$ and Hongbin Wang ${ }^{1 *}$ (D)

\begin{abstract}
Background: The aim of this study was to investigate the association among biofilm formation, virulence gene expression, and antibiotic resistance in P. mirabilis isolates collected from diarrhetic animals $(n=176)$ in northeast China between September 2014 and October 2016.

Results: Approximately $92.05 \%$ of the isolates were biofilm producers, whereas $7.95 \%$ of the isolates were nonproducers. The prevalence of virulence genes in the biofilm producer group was significantly higher than that in the non-producer group. Biofilm production was significantly associated with the expression of ureC, zapA, rsmA, hmpA, mrpA, atfA, and pmfA ( $P<0.05)$. The results of drug susceptibility tests revealed that approximately $76.7 \%$ of the isolates were multidrug-resistant (MDR) and extensively drug-resistant (XDR). Biofilm production was significantly associated with resistance to doxycycline, tetracycline, sulfamethoxazole, kanamycin, and cephalothin $(P<0.05)$. Although the pathogenicity of the biofilm producers was stronger than that of the non-producers, the biofilm-forming ability of the isolates was not significantly associated with morbidity and mortality in mice $(P>0.05)$.

Conclusion: Our findings suggested that a high level of multidrug resistance in P. mirabilis isolates obtained from diarrhetic animals in northeast China. The results of this study indicated that the positive rates of the genes expressed by biofilm-producing P. mirabilis isolates were significantly higher than those expressed by non-producing isolates.
\end{abstract}

Keywords: Proteus mirabilis, Animal, Diarrhea, Biofilm, Virulence gene, Antibiotic resistance, Pathogenicity

\section{Background}

Proteus mirabilis is a motile gram-negative bacillus belonging to the family Enterobacteriaceae. It is an opportunistic pathogen of great importance that is found in water and soil as well as in the intestinal tracts of mammals. It has been recognized as a leading cause of urinary tract infections [1] and the primary infectious factor

\footnotetext{
* Correspondence: hbwang1940@163.com

'College of Veterinary Medicine, Northeast Agricultural University, Harbin 150030, P.R. China

Full list of author information is available at the end of the article
}

in patients with indwelling urinary catheters [2]. P. mirabilis can cause food poisoning, respiratory and wound infections, bacteremia, and other infections [3-6]. In the past decade, diseases associating with $P$. mirabilis infection have also been reported in birds with reproductive failure [7], weaned infant rhesus monkeys (Macaca mulatta) and ferrets (Mustela putorius furo) with diarrhea [8], and dogs with chronic otitis externa [9]. Although P. mirabilis, as an opportunistic pathogen capable of causing serious infections, should not be neglected. 
Various virulence factors contribute to the pathogenicity of $P$. mirabilis. These factors include the presence of fimbriae and specific outer membrane proteins; flagella-based and swarming motility; urease activity;environmental iron binding;cell invasiveness, in addition to lipopolysaccharides and hemolysins, most of which are involved in the ability of bacteria to adhere, colonize, and invade tissues, thereby promoting pathogenicity $[10$, $11]$. Virulence gene expression is not the only factor responsible for the pathogenicity of $P$. mirabilis; biofilm formation exacerbates the complexity of $P$. mirabilis infection [12], as biofilms are recognized as the ultimate cause of persistent and destructive infections and inflammatory processes [13]. A biofilm is an assemblage of microbial cells that adhere to specific surfaces and neighboring cells, and it is covered with an extracellular matrix $[14,15]$. Biofilms inadvertently contribute to bacterial survival, thereby enabling better adaptation to the conditions of the external environment and more effective use of nutrition [16]. P. mirabilis has been found to produce biofilms on a wide range of surfaces, including polystyrene, silicone, latex, glass, and various biological surfaces $[17,18]$. In recent years, studies have reported a correlation between biofilm formation and various virulence factors in P. mirabilis isolates from humans [19, 20]. For instance, catheter encrustation has been reported to be brought about by the activity of ureaseproducing biofilms $[19,21]$. Jansen et al. discovered that mannose-resistant Proteus-like fimbriae produced by $P$. mirabilis, which infects the urinary tract, can induce biofilm formation, with the fimbriae aiding in the aggregation of the bacteria [20]. However, most reports of $P$. mirabilis describe urinary tract infections in humans, and there are few reports on P. mirabilis isolated from diarrhetic animals. In addition, the association between biofilm formation and various virulence factors in $P$. mirabilis isolates from diarrhetic animals is still unknown.

Over the past two decades, due to the identification of multiple multidrug-resistant (MDR) and extensively drug-resistant (XDR) P. mirabilis isolates, the treatment of $P$. mirabilis infections has become increasingly difficult [22-25]. For instance, the production of biofilms by $P$. mirabilis exacerbates the complexity of bacterial resistance, prolongs the treatment time, and further aggravates the infection. In essence, biofilms protect organisms from the host immune system and antimicrobial agents [26]. Furthermore, a previous study has demonstrated that certain antibiotics can induce biofilm formation [27]. However, it is unclear whether antibiotics are linked to biofilm formation by $P$. mirabilis isolates obtained from diarrhetic animals.

Here, we investigate biofilm formation, antimicrobial susceptibility, and virulence gene expression in $P$. mirabilis isolates recovered from feces of various diarrhetic animals in northeast China and discuss the association among the pathogenicity, drug resistance, and virulence of $P$. mirabilis from the perspective of biofilm formation.

\section{Results}

\section{Prevalence of $P$. mirabilis isolates}

The prevalence rates of $P$. mirabilis in the diarrheal samples are listed in Table 1. Overall, 28.66\% (176/614) of diarrheal specimens were positive for $P$. mirabilis. The positive isolation rate of $P$. mirabilis in different species was less than $40 \%$. The positive rates of $P$. mirabilis isolated from specimens was $32.76 \%$ for dog, followed by $28.7 \%$ for mink, $23.26 \%$ for cattle, and $22.5 \%$ for fowl.

\section{Biofilm formation}

Of the P. mirabilis isolates $(n=176)$ tested, $162(92.05 \%)$ were biofilm producers and $14(7.95 \%)$ were nonproducers. Of the biofilm producers $(n=162), 78$ $(48.15 \%)$ were moderate biofilm producers, whereas 62 (38.27\%) and 22 (13.58\%) were strong and weak biofilm producers, respectively.

\section{Virulence gene expression}

The prevalence rates of ureC, zapA, rsmA, hmpA, mrpA, atfA, pmfA, FliL, and ucaA in P. mirabilis are listed in Table 2. Of the $P$. mirabilis isolates, the most prevalent gene was $u r e C$, which was identified in $90.91 \%$ of the isolates, followed by zapA, $r \operatorname{sm} A, \operatorname{hmp} A, \operatorname{mrp} A$, atfA, pmfA, FliL, and ucaA, which were detected in 85.8, $81.25,70.45,65.91,64.77,60.23,56.82$, and $32.95 \%$ of the isolates, respectively. The positive rates of genes identified in the biofilm-producing isolates were significantly higher than those in the non-producing isolates. Furthermore, except for hmpA,eight genes tested in the moderate biofilm producing isolates were higher than those in the strong and weak biofilm producing isolates. All nine genes tested in this study showed the lowest prevalence rates among the non-producers. Biofilm production was significantly associated with the expression of ureC, zapA, rsmA, hmpA, mrpA, atfA, and pmfA $(P<$ 0.05).

Table 1 Prevalence of P. mirabilis in collected diarrheal samples

\begin{tabular}{lll}
\hline Host & Number of samples & Number of positive samples (\%) \\
\hline Dog & 232 & $76(32.76)$ \\
Mink & 216 & $62(28.7)$ \\
Cattle & 86 & $20(23.26)$ \\
Fowl & 80 & $18(22.5)$ \\
Total & 614 & $176(28.66)$ \\
\hline
\end{tabular}


Table 2 Prevalence of ureC, zapA, rsmA, hmpA, mrpA, atfA, pmfA, FliL and ucaA genes in P. mirabilis isolates

\begin{tabular}{|c|c|c|c|c|c|}
\hline \multirow{2}{*}{$\begin{array}{l}\text { Virulence } \\
\text { gene }\end{array}$} & \multicolumn{5}{|l|}{ P. mirabilis } \\
\hline & $\begin{array}{l}\text { High biofilm producer } \\
n=62\end{array}$ & $\begin{array}{l}\text { Moderate biofilm producer } \\
n=78\end{array}$ & $\begin{array}{l}\text { Weak biofilm producer } \\
n=22\end{array}$ & $\begin{array}{l}\text { Non biofilm producer } \\
n=14\end{array}$ & $P$ value \\
\hline ureC & $58(93.54 \%)$ & $76(97.43 \%)$ & $16(72.72 \%)$ & $10(71.43 \%)$ & $P<0.0001$ \\
\hline zapA & $52(83.87 \%)$ & $72(92.31 \%)$ & $15(68.18 \%)$ & $12(85.71 \%)$ & 0.037 \\
\hline $\operatorname{rsm} A$ & $50(80.64 \%)$ & $70(89.74 \%)$ & $14(63.64 \%)$ & $9(64.29 \%)$ & 0.013 \\
\hline hmpA & 48 (77.42\%) & $60(76.92 \%)$ & $10(45.45 \%)$ & $6(42.85 \%)$ & 0.002 \\
\hline mrpA & $41(66.13 \%)$ & $65(83.33 \%)$ & $8(36.36 \%)$ & $2(14.29 \%)$ & $P<0.0001$ \\
\hline atfA & $40(64.52 \%)$ & $62(79.49 \%)$ & $10(45.45 \%)$ & $2(14.29 \%)$ & $P<0.0001$ \\
\hline pmfA & $38(61.29 \%)$ & $58(74.36 \%)$ & $8(36.36 \%)$ & $2(14.29 \%)$ & $P<0.0001$ \\
\hline Flil & $33(53.23 \%)$ & $50(64.1 \%)$ & $10(45.45 \%)$ & $7(50 \%)$ & 0.329 \\
\hline UCaA & $14(22.58 \%)$ & $32(41.03 \%)$ & 7 (31.82\%) & $5(35.71 \%)$ & 0.146 \\
\hline
\end{tabular}

\section{Antimicrobial susceptibility testing}

The antimicrobial resistance patterns of the P. mirabilis isolates are shown in Fig. 1. Variable degrees of resistance of these isolates to all antibiotics tested were observed. The resistance to doxycycline was the highest (112, 63.64\%), followed by ampicillin (104, 59.09\%), ciprofloxacin (101, 57.39\%), streptomycin $(98,55.68 \%)$, tetracycline $(97,55.12 \%)$, piperacillin/tazobactam (88, $50 \%)$, cefotaxime $(86,48.87 \%)$, sulfamethoxazole (76, $43.19 \%)$, nitrofurantoin $(75,42.61 \%)$, polymyxin B (69, $39.2 \%)$, ceftriaxone $(67,38.07 \%)$, kanamycin (67, $38.07 \%)$, ceftazidime $(62,35.23 \%)$, gentamicin (60, $34.09 \%)$, cephalothin $(53,30.12 \%)$, cefoperazone (50, $28.41 \%)$, levofloxacin $(45,25.57 \%)$, meropenem (44, $25 \%)$, and imipenem $(36,20.45 \%)$. Of the sensitive strains, meropenem (57.96\%) and imipenem (64.78\%) showed the strongest antimicrobial effect on $P$. mirabilis (Fig. 1a).

The MDR patterns of the $P$. mirabilis isolates are shown in Fig. 1b. No isolate was sensitive to all the antibiotics. Of the P. mirabilis isolates, 18 and 23 isolates were resistant to only one or two of the 19 antibiotics tested, respectively, and 106 isolates were MDR, whereas 29 isolates were XDR. Thus, approximately $76.7 \%$ of the strains exhibited MDR or XDR.

The antibiotic susceptibility patterns of the biofilm producing and non-producing $P$. mirabilis isolates are shown in Table 3. Both biofilm producers and nonproducers were highly resistant to doxycycline and moderately resistant to cefotaxime. Of the 19 antibiotics, doxycycline, ampicillin, tetracycline, cefotaxime, and kanamycin were found to be non-susceptible to nonproducers. A sensitivity of $62.95 \%$ was observed for the biofilm-producing isolates to imipenem, whereas a sensitivity of $85.71 \%$ was noticed for the non-producing isolates to meropenem and imipenem. Isolates showing sensitivity to doxycycline, ampicillin, ciprofloxacin, streptomycin, tetracycline, piperacillin/tazobactam, cefotaxime, nitrofurantoin, sulfamethoxazole, and ceftriaxone produced more biofilm than strains showing resistance to these antibiotics. For other antibiotics, such as polymyxin B, ceftazidime, kanamycin, gentamicin, cefoperazone, cephalothin, meropenem, levofloxacin, and imipenem, we observed opposite findings. Biofilm production was significantly associated with resistance to doxycycline, tetracycline, sulfamethoxazole, kanamycin, and cephalothin $(P<0.05)$.

\section{Pathogenicity test in mice}

The morbidity and mortality of mice that received 32 different isolates of pathogenic bacteria is demonstrated in Table 4. Mice in the negative control group were obviously asymptomatic and in good overall health. Within $12 \mathrm{~h}$ of challenge, different symptoms of variable degrees were observed in mice of the experimental groups. These symptoms included gloomy spirit, inactivity, and loss of appetite. In the biofilm-producing group, some mice died within $12 \mathrm{~h}$ of challenge. Mice in both the biofilm producing and non-producing groups showed severe clinical symptoms, such as diarrhea, abrosia, subdued behavior, hunched appearance, and absence of grooming within $24 \mathrm{~h}$ of challenge. Eleven mice $(45.83 \%)$ in the biofilm producing and non-producing groups died within $48 \mathrm{~h}$ of challenge. After $72 \mathrm{~h}$ of challenge, the symptoms began to gradually disappear, and the health of most mice returned to normal. Only two mice (25\%) in the non-producing group died within $72 \mathrm{~h}$ of challenge. The biofilm-forming ability of $P$. mirabilis was not significantly associated with morbidity and mortality in mice $(P>0.05)$.

\section{Discussion}

Biofilm formation by $P$. mirabilis has recently become an issue of increasing concern. In a previous study, $P$. mirabilis isolates recovered from urine samples showed a higher degree of biofilm production than those isolated 


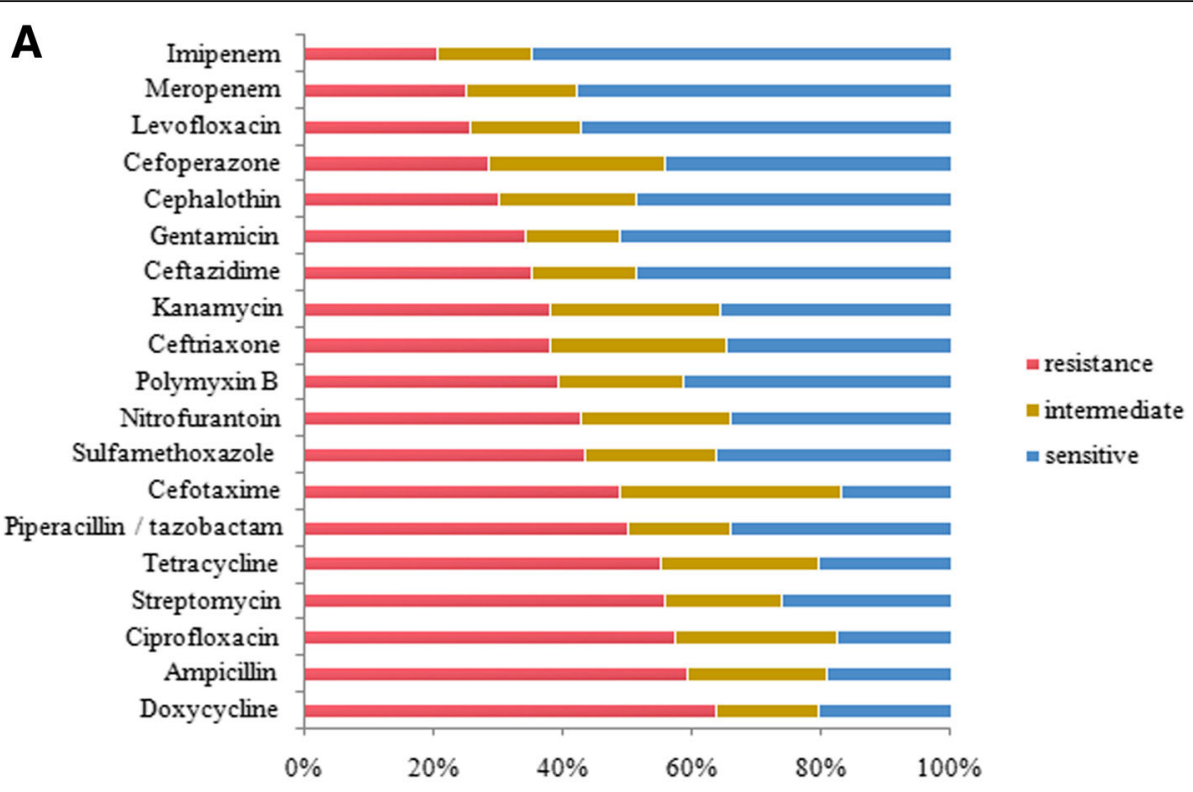

B

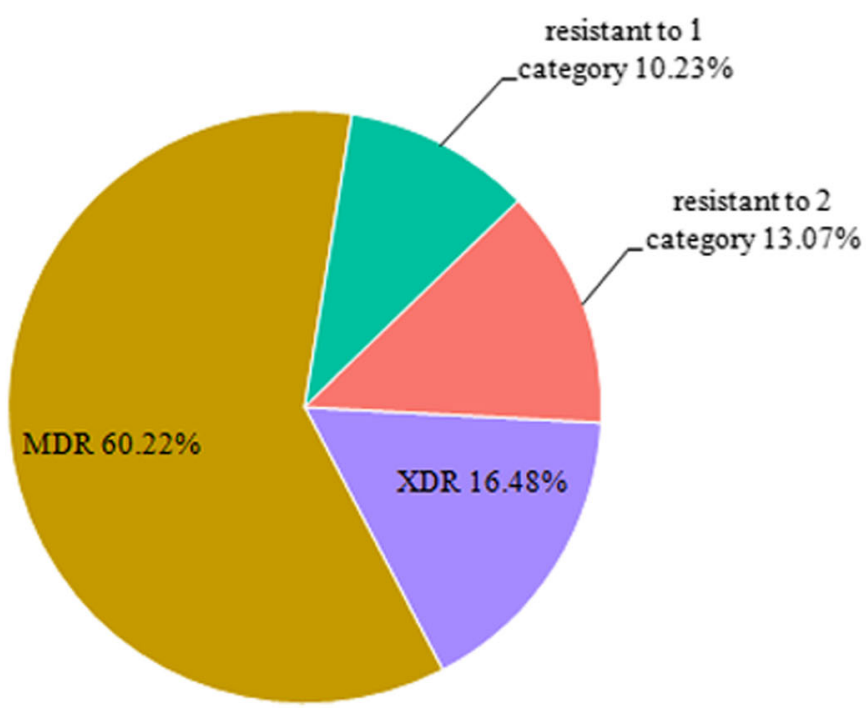

Fig. 1 Antibiotic resistance phenotypes of $P$. mirabilis isolates examined in this study. a Resistance rates of all isolates to 19 antibiotics. b Approximately $76.7 \%$ of the isolates exhibited multidrug or extensive drug resistance

from different catheter segments [28]. In this study, the prevalence of $P$. mirabilis was high, which is in agreement with the results of studies on urine from catheterized patients $[12,28]$. Taken collectively, these findings indicate that further studies on $P$. mirabilis biofilm formation are needed to better understand the disease process and to develop new preventive and therapeutic options.

As a target gene, ureC, was used to positively identify $P$. mirabilis as described previously [29]. Ali et al. reported that $96.66 \%$ of human $P$. mirabilis isolates $(n=$ 30) recovered from the urinary tract expressed ureC [30]. In this study, $90.91 \%$ of the isolates were positive for ureC. However, its prevalence was relatively low when compared to the results of an earlier study [29]. Our findings revealed that testing only $u r e C$ increased the likelihood of obtaining $P$. mirabilis negative results. Therefore, we used a PCR method based on $16 S$ rRNA expression to detect $P$. mirabilis. The results of our study showed that the prevalence rates of zapA, $\operatorname{rsm} A$, $h m p A, m r p A$, atfA, pmfA, FliL, and ucaA were relatively high. Furthermore, the overall prevalence of $P$. mirabilis was much higher in our study than that in previous studies [31-36].

The prevalence rates of eight genes expressed by moderate biofilm producers were significantly higher than 
Table 3 Antibiotic resistance pattern of the biofilm producing and non-producing P. mirabilis isolates

\begin{tabular}{|c|c|c|c|c|c|c|c|}
\hline \multirow[b]{2}{*}{ Antibiotic } & \multicolumn{3}{|c|}{ Biofilm producer $(n=162)$} & \multicolumn{4}{|c|}{ Non biofilm producer $(n=14)$} \\
\hline & resistance & intermediate & sensitive & resistance & intermediate & sensitive & $P$ value \\
\hline Doxycycline & $98(60.49 \%)$ & $28(17.28 \%)$ & $36(22.23 \%)$ & $14(100 \%)$ & $0(0 \%)$ & $0(0 \%)$ & 0.015 \\
\hline Ampicillin & $92(56.79 \%)$ & $36(22.23 \%)$ & $34(20.98 \%)$ & $12(85.71 \%)$ & $2(14.29 \%)$ & $0(0 \%)$ & 0.078 \\
\hline Ciprofloxacin & $90(55.56 \%)$ & $44(27.16 \%)$ & $28(17.28 \%)$ & $11(78.57 \%)$ & $0(0 \%)$ & $3(21.43 \%)$ & 0.084 \\
\hline Streptomycin & $88(54.32 \%)$ & $30(18.52 \%)$ & $44(27.16 \%)$ & $10(71.42 \%)$ & $2(14.29 \%)$ & $2(14.29 \%)$ & 0.445 \\
\hline Tetracycline & $85(52.47 \%)$ & $41(25.3 \%)$ & $36(22.23 \%)$ & $12(85.71 \%)$ & $2(14.29 \%)$ & $0(0 \%)$ & 0.034 \\
\hline Piperacillin/tazobactam & $84(51.85 \%)$ & $26(16.05 \%)$ & $52(32.1 \%)$ & $4(28.58 \%)$ & $2(14.29 \%)$ & $8(57.13 \%)$ & 0.15 \\
\hline Cefotaxime & $78(48.15 \%)$ & $54(33.33 \%)$ & $30(18.52 \%)$ & $8(57.13 \%)$ & $6(42.87 \%)$ & $0(0 \%)$ & 0.255 \\
\hline Nitrofurantoin & $67(41.36 \%)$ & $39(24.07 \%)$ & $56(34.57 \%)$ & $8(57.13 \%)$ & 2 (14.29\%) & $4(28.58 \%)$ & 0.492 \\
\hline sulfamethoxazole & 64 (39.5\%) & $36(22.23 \%)$ & $62(38.27 \%)$ & 12 (85.71\%) & $0(0 \%)$ & $2(14.29 \%)$ & 0.004 \\
\hline Ceftriaxone & $62(38.27 \%)$ & $45(27.78 \%)$ & 55 (33.95\%) & $5(35.7 \%)$ & $3(21.43 \%)$ & $6(42.87 \%)$ & 0.776 \\
\hline Polymyxin B & $61(37.66 \%)$ & 32 (19.75\%) & 69 (42.59\%) & $8(57.13 \%)$ & $2(14.29 \%)$ & $4(28.58 \%)$ & 0.357 \\
\hline Ceftazidime & $58(35.8 \%)$ & $28(17.28 \%)$ & 76 (46.92\%) & 4 (28.58\%) & $0(0 \%)$ & 10 (71.42\%) & 0.131 \\
\hline Kanamycin & 57 (35.19\%) & $42(25.93 \%)$ & 63 (38.88\%) & $10(71.42 \%)$ & $4(28.58 \%)$ & $0(0 \%)$ & 0.005 \\
\hline Gentamicin & $52(32.1 \%)$ & $24(14.82 \%)$ & $86(53.08 \%)$ & $8(57.13 \%)$ & 2 (14.29\%) & $4(28.58 \%)$ & 0.143 \\
\hline Cefoperazone & $48(29.63 \%)$ & 46 (28.4\%) & 68 (41.97\%) & 2 (14.29\%) & 2 (14.29\%) & 10 (71.42\%) & 0.104 \\
\hline Cephalothin & $45(27.78 \%)$ & 37 (22.84\%) & $80(49.38 \%)$ & $8(57.13 \%)$ & $0(0 \%)$ & $6(42.87 \%)$ & 0.030 \\
\hline Meropenem & $42(25.93 \%)$ & $30(18.52 \%)$ & $90(55.55 \%)$ & 2 (14.29\%) & $0(0 \%)$ & 12 (85.71\%) & 0.061 \\
\hline Levofloxacin & 39 (24.07\%) & $30(18.52 \%)$ & $93(57.41 \%)$ & $6(42.87 \%)$ & $0(0 \%)$ & $8(57.13 \%)$ & 0.117 \\
\hline Imipenem & $36(22.23 \%)$ & $24(14.82 \%)$ & 102 (62.95\%) & $0(0 \%)$ & 2 (14.29\%) & 12 (85.71\%) & 0.114 \\
\hline
\end{tabular}

those expressed by strong and weak biofilm producers. These results show that moderate biofilm producers are highly virulent. This is also the first study reporting that the biofilm-forming ability of $P$. mirabilis is significantly associated with the expression of ureC, zapA, rsmA, $h m p A, m r p A$, atfA, and $p m f A(P<0.05)$. Biofilm formation is associated with the adhesion and aggregation of bacteria [20], and $\operatorname{rsm} A, \operatorname{mrp} A$, and atfA have been reported to be involved in bacterial adhesion and aggregation [31-33], which is consistent with the results of this study. Biofilm formation was also associated with ureC expression, as previously reported $[19,21]$. However, the association between $z a p A$ and $h m p A$, and biofilm formation needs further study.

The results of antimicrobial susceptibility tests revealed high resistance rates of the $P$. mirabilis isolates to several antibiotics such as doxycycline and ampicillin. The resistance rates ranged from 30 to $50 \%$. The $P$.

Table 4 Pathogenicity to mice in 32 biofilm producing and non-producing $P$. mirabilis isolates from animal with diarrhea

\begin{tabular}{lccc}
\hline Pathogenicity & $\begin{array}{l}P \text {. mirabilis } \\
\text { Biofilm producer } \\
(n=24)\end{array}$ & $\begin{array}{l}\text { Non biofilm producer } \\
(n=8)\end{array}$ & $P$ value \\
\hline morbidity & $17(70.83 \%)$ & $5(62.5 \%)$ & 0.660 \\
mortality & $11(45.83 \%)$ & $2(25 \%)$ & 0.299 \\
\hline
\end{tabular}

mirabilis isolates that were resistant to antibiotics were similar to those previously isolated from chicken products in Hong Kong [37]. However, the resistance rates of $P$. mirabilis to streptomycin, sulfamethoxazole, kanamycin, ampicillin, ciprofloxacin, cephalothin, gentamicin, cefotaxime, and ceftazidime were significantly higher than those reported in an earlier study, which isolated $P$. mirabilis from dogs [38]. Our results on the resistance rates of $P$. mirabilis to nitrofurantoin, tetracycline, and polymyxin $B$ were much lower than those reported in a human study [39], although the resistant rates to other antibiotics, except nitrofurantoin, tetracycline, and polymyxin $\mathrm{B}$, were generally high $[39,40]$. In a previous study, $P$. mirabilis isolates from dogs were found to be highly sensitive to ciprofloxacin and gentamicin [41]. In this study, meropenem and imipenem were the most effective antibiotics against $P$. mirabilis, which is consistent with the results of an earlier study [42]. Meropenem and imipenem are classified as carbapenems, and our results showed that carbapenems were highly effective against $P$. mirabilis. We also found that approximately $76.7 \%$ of the isolates were MDR or XDR, which is very high compared to the rate reported in a previous study [43]. This finding reveals that resistance to $P$. mirabilis was steadily increasing. It is also important to mention that the high number of MDR P. mirabilis isolates obtained from companion animals may pose a potential 
threat to human health. Further studies are needed to define the mechanism of resistance, which may improve the treatment of $P$. mirabilis infections in the future.

In this study, we found that the resistance to several antibiotics, including piperacillin/tazobactam, ceftazidime, cefoperazone, ceftriaxone, meropenem, and imipenem, was significantly higher among biofilm producers than non-producers, indicating that biofilm producers were more resistant to antibiotics than non-producers. Presently, few studies have described an association between biofilm formation and drug resistance of $P$. mirabilis, although similar studies have been performed for other pathogens such as uropathogenic E. coli $[44,45]$, coagulase-negative staphylococci [46], and Haemophilus parasuis [47]. Except for piperacillin/tazobactam, ceftazidime, cefoperazone, ceftriaxone, meropenem, and imipenem, the antibiotic resistance rates of non-producers were higher than those of producers. This finding is consistent with an earlier study that reported non-MDR Acinetobacter baumannii isolates to participate in robust biofilm formation [27]. Different resistance mechanisms are likely to be responsible for the differences in antibiotic resistance and biofilm formation in various bacteria. We also found that the biofilm-forming ability of the $P$. mirabilis isolates was significantly associated with the resistance to doxycycline, tetracycline, sulfamethoxazole, kanamycin, and cephalothin $(P<0.05)$. $\beta$-lactamase has been reported to decrease the ability of E. coli to form biofilms by inhibiting peptidoglycans, which are required for the assembly of surface molecules on the biofilm. Under sub-inhibitory concentrations of tetracycline and ampicillin, the overexpression of the TetA $(C)$ pump, which contributes to the osmotic stress response and induces capsular colanic acid production, promoted the formation of mature biofilms [48]. In addition, there was an association between the resistance to aminoglycoside and extracellular DNA in Pseudomonas aeruginosa [49]. Thus, we hypothesize that antibiotic resistance is associated with the composition of $P$. mirabilis biofilms, thereby affecting biofilm formation.

Although the pathogenicity of the biofilm producers was stronger than that of the non-producers, the biofilm-forming ability of the isolates was not significantly associated with morbidity and mortality in mice $(P>0.05)$. The high pathogenicity of the biofilm producers may have been due to the fact that the biofilmforming ability of $P$. mirabilis associated with the expression of ureC, zapA, rsmA, hmpA, $m r p A$, atfA, and pmfA $(P<0.05)$. Furthermore, it has been reported that ure $C$ and zapA expressed by $P$. mirabilis associate with diarrhea in goats [50]. Gabidullin et al. found that diarrhea caused by $P$. mirabilis was related to enterotoxins [51]. Thus, the mechanism responsible for $P$. mirabilisinduced diarrhea needs further study.

\section{Conclusions}

In conclusion, our study revealed a high level of multidrug resistance in $P$. mirabilis isolates recovered from diarrhetic animals in northeast China. Therefore, the use of antimicrobial agents in animals needs to be controlled so as to minimize the emergence and eventual spread of resistant pathogens, which is warranted in order to protect human health. The results of this study indicated that the positive rates of the genes expressed by biofilmproducing $P$. mirabilis isolates were significantly higher than those expressed by non-producing isolates. Furthermore, this is the first study to report that the biofilmforming ability of $P$. mirabilis isolates from diarrhetic animals is significantly associated with the expression of ureC, zapA, rsmA, hmpA, $m r p A$, atfA, and $p m f A$ and the resistance to doxycycline, tetracycline, sulfamethoxazole, kanamycin, and cephalothin. Further studies on biofilm formation by $P$. mirabilis are necessary to better understand the disease process and to develop effective treatments for mammals with antibiotic resistant $P$. mirabilis.

\section{Methods \\ Sample collection}

Six hundred and fourteen fecal swabs were collected randomly from different farms in northeast China between September 2014 and October 2016 and transferred to the laboratory in ice-filled containers. Fecal swabs were collected from different animals (i.e., dog, mink, cattle, and fowl) with diarrhea.

\section{P. mirabilis screening}

P. mirabilis was isolated as described previously [37]. To grow the bacteria, the fecal swabs were incubated with 1 $\mathrm{mL}$ of Luria-Bertani broth for $6 \mathrm{~h}$ at $37^{\circ} \mathrm{C}$, and a $30 \mu \mathrm{L}$ aliquot of the broth was added to buffered peptone water (Aobox, Beijing, China). The resultant mixture was incubated for $24 \mathrm{~h}$ at $37^{\circ} \mathrm{C}$ to enrich the culture, and then streaked onto xylose lysine deoxycholate agar plates (Hopebiol, Qingdao, China). All plates were incubated at $37^{\circ} \mathrm{C}$ for a minimum of $16 \mathrm{~h}$. One isolate from each sample was selected for further characterization.

\section{Identification of isolates}

To identify $P$. mirabilis isolates, standard biochemical tests were used as described previously [52]. The isolates were gram-negative bacilli that were positive for glucose, methyl red, and urease but negative for maltose, sucrose, mannitol, indole, and gelatin liquefaction. The VogesProskauer test yielded negative results for the isolates. Urease $\mathrm{C}$ (ureC) and mannose-resistant Proteus-like fimbriae $(m r p A)$ were amplified by the polymerase chain reaction (PCR) to identify $P$. mirabilis from the presumptive isolates [36]. Positive results were randomly selected for sequencing. When the results were negative 
for ure $C$ and $m r p A$ expression, a segment of the $16 S$ $r R N A$ gene was amplified. The $16 S$ rRNA segment was sequenced [53]. The sequences were compared with ureC, $m r p A$, and $16 S$ rRNA in GenBank using BLAST.

\section{Quantification of biofilm production}

Biofilm production was assessed using a microtiter plate assay as described previously [54]. P. mirabilis isolates were grown in tryptic soy broth (Hopebiol) supplemented with $0.25 \%$ glucose overnight at $37^{\circ} \mathrm{C}$. Subsequently, the cultures were diluted to $1 \times 10^{6} \mathrm{CFU} \mathrm{mL}^{-1}$ in fresh tryptic soy broth supplemented with $0.25 \%$ glucose, and $200 \mu \mathrm{L}$ of the diluted culture was transferred into each well of a sterile flat-bottom 96 -well polystyrene microtiter plate. After incubation for $24 \mathrm{~h}$ at $37^{\circ} \mathrm{C}$ without shaking, the wells were gently washed three times with $200 \mu \mathrm{L}$ of distilled water. The biofilms were fixed with $200 \mu \mathrm{L}$ of $99 \%$ methanol for $15 \mathrm{~min}$. The supernatant was removed, and the plate was air-dried. The biofilms were then stained with $200 \mu \mathrm{L}$ of $1 \%$ crystal violet for $10 \mathrm{~min}$. The excess dye was removed by washing the plate under running tap water. Finally, the bound crystal violet was released by adding $200 \mu \mathrm{L}$ of $33 \%$ acetic acid. The optical density (OD) of each well was measured at $590 \mathrm{~nm}$ using a microtiter plate reader. To determine the background OD, control experiments were performed with uninoculated broth. This assay was repeated three independent times. The biofilm formation capacity of each isolate was analyzed using the method of Khoramian et al. [55].

\section{Expression of virulence genes}

$P$. mirabilis virulence genes were detected by PCR. These genes were ure $C$, extracellular metalloprotease $(z a p A)$, swarming behavior $(\operatorname{rsm} A)$, hemolysin $($ hpmA), $m r p A$, ambient-temperature fimbriae (atfA), P. mirabilis fimbriae ( $p m f A)$, flagellar basal body protein $(F l i L)$, and uroepithelial cell adhesin fimbriae (ucaA) (Table 5) [10]. The base sequences and predicted sizes of the PCR products for the specific oligonucleotide primers used in this study are listed in Table 5. Subsequently, total genomic DNA was isolated from stationary-phase broth cultures that were grown overnight in Luria-Bertani broth with the TIANamp Bacterial DNA Kit (TIANGEN, Beijing, China) according to the manufacturer's instructions. All PCR products were analyzed by agarose gel electrophoresis, and the results were recorded with a gel documentation system. All amplifications were repeated three independent times in parallel with a negative control (distilled water served as the PCR template).

Table 5 Primers used in the PCRs carried out in this study

\begin{tabular}{|c|c|c|c|c|c|}
\hline Target gene & Primer & Nucleotide Sequence (5'-3') & Amplicon (bp) & $\mathrm{AT}^{*}$ & Reference \\
\hline \multirow[t]{2}{*}{$16 S r R N A$} & $27 \mathrm{~F}$ & AGAGTTTGATCCTGGCTCAG & 1463 & 49 & Leite et al. (2015) [53] \\
\hline & $1492 \mathrm{R}$ & GGTTACCTTGTTACGACTT & & & \\
\hline \multirow[t]{2}{*}{ urec } & ureC-F & GTTATTCGTGATGGTATGGG & 317 & 52 & Stankowska et al. (2008) [36] \\
\hline & ureC-R & ATAAAGGTGGTTACGCCAGA & & & \\
\hline \multirow[t]{2}{*}{$z a p A$} & zapA-F & ACCGCAGGAAAACATATAGCCC & 540 & 53 & Stankowska et al. (2008) [36] \\
\hline & $z a p A-R$ & GCGACTATCTTCCGCATAATCA & & & \\
\hline \multirow[t]{2}{*}{$r s m A$} & $r s m A-F$ & TAGCGAGTGTTGACGAGTGG & 562 & 49 & Shi et al. (2016) [31] \\
\hline & rsmA-R & AGCGAGGTGAAGAACGAGAA & & & \\
\hline \multirow[t]{2}{*}{ hpmA } & hpmA-F & CCAGTGAATTAACGGCAGGT & 654 & 49 & Shi et al. (2016) [31] \\
\hline & hpmA-R & CGTGCCCAGTAATGGCTAAT & & & \\
\hline \multirow[t]{3}{*}{ mrpA } & MRP-F & ACACCTGCCCATATGGAAGATACTGGTACA & 550 & 40 & Barbour et al. (2012) [32] \\
\hline & $M R P-R$ & AAGTGATGAAGCTTAGTGATGGTGATGGTG & & & \\
\hline & & ATGAGAGTAAGTCACC & & & \\
\hline \multirow[t]{2}{*}{ FliL } & FliL-F & CTCTGCTCGTGGTGGTGTCG & 770 & 40 & Barbour et al. (2012) [32] \\
\hline & FliL-R & GCGTCGTCACCTGATGTGTC & & & \\
\hline \multirow[t]{2}{*}{ ucaA } & ucaA-F & GTAAAGTTGTTGCGCAAAC & 560 & 50 & Sosa et al. (2006) [35] \\
\hline & UCAA-L & TTGAGCCACTGTGGATACA & & & \\
\hline \multirow[t]{2}{*}{ pmfA } & pmfA-F & CAAATTAATCTAGAACCACTC & 618 & 54 & Zunino et al. (2003) [34] \\
\hline & pmfA-R & ATTATAGAGGATCCCTTGAAGGTA & & & \\
\hline \multirow[t]{2}{*}{ atfA } & atfa-F & CATAATTTCTAGACCTGCCCTAGCA & 382 & 50 & Zunino et al. (2000) [33] \\
\hline & $a t f A-R$ & CTGCTTGGATCCGTAATTITTAACG & & & \\
\hline
\end{tabular}




\section{Antimicrobial resistance test}

The antimicrobial resistance of the isolates to 19 antibiotics, including doxycycline, ampicillin, ciprofloxacin, streptomycin, tetracycline, piperacillin/tazobactam, cefotaxime, nitrofurantoin, sulfamethoxazole, ceftriaxone, polymyxin $\mathrm{B}$, ceftazidime, kanamycin, gentamicin, cefoperazon, cephalothin, meropenem, levofloxacin, and imipenem (BIO-KONT, Wenzhou, China), was determined by the Kirby-Bauer disc diffusion method [56]. In brief, 0.5 McFarland P. mirabilis inoculum was spread onto Mueller-Hinton agar plates (Hopebiol), and the antibiotic discs were dispensed on the agar. The plates were incubated at $37^{\circ} \mathrm{C}$ for $18 \mathrm{~h}$. Escherichia coli (E. coli) ATCC 25922 was used as the control microorganism. The inhibitory zone around each disc was measured, and the results were interpreted according to the guidelines provided by the manufacturers and the Clinical and Laboratory Standards Institute [57]. The results were interpreted as resistant, intermediate, and susceptible. MDR was defined as acquired non-susceptibility to at least one agent in three or more antimicrobial categories. XDR was defined as non-susceptibility to at least one agent in all but two or fewer antimicrobial categories [58]. Non-MDR was defined as resistance to none or up to two antimicrobial categories [27].

\section{Pathogenicity test in mice}

The pathogenicity test was performed as described previously [59]. In brief, healthy, 6-week-old, female BALB/C mice $(n=99)$ (Changsheng Biotechnology Co., Ltd., Liaoning, China) were used to investigate the pathogenicity of $P$. mirabilis. The mice were randomly divided into thirtythree groups ( $n=3 /$ per group) as follows: one control group and thirty-two experimental groups, which tested $32 \mathrm{P}$. mirabilis isolates for their pathogenicity to produce biofilm, to express various virulence genes, and to resist different drugs. Each mouse received a 0.2-mL intraperitoneal injection of a bacterial suspension at a concentration of $1 \times 10^{8} \mathrm{CFU} \mathrm{mL} \mathrm{m}^{-1}$, and the control mice were challenged with vehicle alone. All the mice were fed normally and observed daily for the activity level and water intake for up to 14 days, and deaths were recorded,then the mice alive were euthanized by intraperitoneal injection of 40 $\mathrm{mg} / \mathrm{kg}$ b.w. of sodium thiopental [60].

\section{Statistical analysis}

Data were analyzed with SPSS 18.0 Software (SPSS Statistics, Inc., Chicago, IL, USA). The Chi-square test was adopted for analysis. $P$-values $<0.05$ were considered statistically significant.

\section{Abbreviations}

P. mirabilis: Proteus mirabilis; PCR: Polymerase chain reaction; MDR: Multidrug resistant; XDR: Extensively drug-resistant; BPW: Buffered peptone water; OD: Optical density; E. coli: Escherichia coli; AT: Annealing temperature

\section{Acknowledgments}

The authors thank Dr. Songfeng Zheng (Missouri State University, USA) for his help with statistical analysis.

\section{Authors' contributions}

All authors participated in study design and planning. YDS and SSW conceived and designed the study as well as acquired and extracted the data. YDS performed data analysis. YDS, SSW, LLZ, Y P, and QQX drafted the manuscript. YDS, SSW, HHL, CWW, HYC, JWG, and HBW interpreted the data and revised the manuscript. All authors have read and approved this version of the manuscript for publication.

\section{Funding}

This work was supported by the "Academic Backbone" Project of Northeast Agricultural University (17XG10), the National Natural Science Foundation of China (31672532), and the SIPT Program (201910224139). The role of the funding bodies was to pay for the consumables used in this study. They had no role in the design of the study; the collection, analysis, and interpretation of data and in the writing of the manuscript.

\section{Availability of data and materials}

The datasets generated and/or analysed during the current study are available in National Center for Biotechnology Information (NCBI) repository, under these GenBank accession numbers MT294143-MT294148 and MT276297-MT276312.

\section{Ethics approval and consent to participate}

This study was performed in accordance with the Guide for the Care and Use of Laboratory Animals of the Ministry of Health, China. The protocol was reviewed and approved by the Institutional Animal Care and Use Committee of Northeast Agricultural University (2014-SRM-24). The specimens were collected from animals for laboratory analyses only, and unnecessary pain and suffering of the animals was avoided. The animal owners provided written consent for the collection of specimens. Animal experiments were carried out according to animal welfare standards.

\section{Consent for publication}

Not applicable.

\section{Competing interests}

The authors have no financial or personal relationships with other individuals or organizations that could have inappropriately influenced or biased the contents of this paper.

\section{Author details}

${ }^{1}$ College of Veterinary Medicine, Northeast Agricultural University, Harbin 150030, P.R. China. 'Liaoning Vocational College of Ecological Engineering, Shenyang 110122, P.R. China. ${ }^{3}$ State Key Laboratory of Veterinary Biotechnology, Heilongjiang Provincial Key Laboratory of Laboratory Anima and Comparative Medicine, Harbin Veterinary Research Institute, Chinese Academy of Agricultural Sciences, Harbin 150069, P.R. China. ${ }^{4}$ Northeastern Science Inspection Station, China Ministry of Agriculture Key Laboratory of Animal Pathogen Biology, Harbin 150030, P.R. China.

Received: 29 September 2019 Accepted: 12 May 2020

Published online: 05 June 2020

References

1. Chen CY, Chen YH, Lu PL, Lin WR, Chen TC, Lin CY. Proteus mirabilis urinary tract infection and bacteremia: risk factors, clinical presentation, and outcomes. J Microbiol Immunol Infect. 2012;45:228-36.

2. Liu MC, Lin SB, Chien HF, Wang WB, Yuan YH, Hsueh PR, et al. 10'(Z), 13'(E)heptadecadienylhydroquinone inhibits swarming and virulence factors and increases polymyxin B susceptibility in Proteus mirabilis. PLoS One. 2012;7: e45563.

3. Mobley HL, Belas R. Swarming and pathogenicity of Proteus mirabilis in the urinary tract. Trends Microbiol. 1995:3:280-4

4. Wang Y, Zhang S, Yu J, Zhang H, Yuan Z, Sun Y, et al. An outbreak of Proteus mirabilis food poisoning associated with eating stewed pork balls in brown sauce, Beijing. Food Control. 2010;21:302-5. 
5. Wang JT, Chen PC, Chang SC, Shiau YR, Wang HY, Lai JF, et al. Antimicrobial susceptibilities of Proteus mirabilis: a longitudinal nationwide study from the Taiwan surveillance of antimicrobial resistance (TSAR) program. BMC Infect Dis. 2014;14:486.

6. Habibi M, Karam MRA, Bouzari S. Construction and evaluation of the immune protection of a recombinant divalent protein composed of the MrpA from MR/P fimbriae and flagellin of Proteus mirabilis strain against urinary tract infection. Microb Pathog. 2018;117:348-55.

7. Cabassi C, Taddei S, Predari G, Galvani G, Ghidini F, Schiano E, et al. Bacteriologic findings in ostrich (Struthio camelus) eggs from farms with reproductive failures. Avian Dis. 2004;48:716-22.

8. Yu W, He Z, Huang F. Multidrug-resistant Proteus mirabilis isolated from newly weaned infant rhesus monkeys and ferrets. Jundishapur J Microbiol. 2015;8:e16822. https://doi.org/10.5812/jjm.8(5)2015.16822

9. Malayeri $\mathrm{HZ}$, Jamshidi S, Salehi TZ. Identification and antimicrobial susceptibility patterns of bacteria causing otitis externa in dogs. Vet Res Commun. 2010;34:435-44.

10. Rózalski A, Sidorczyk Z, Kotełko K. Potential virulence factors of Proteus bacilli. Microbiol Mol Biol Rev. 1997;61:65-89.

11. Legnani-Fajardo C, Zunino P, Piccini C, Allen A, Maskell D. Defined mutants ofProteus mirabilislacking flagella cause ascending urinary tract infection in mice. Microb Pathog. 1996;21:395-405.

12. Hola V, Peroutkova T, Ruzicka F. Virulence factors in Proteus bacteria from biofilm communities of catheter-associated urinary tract infections. FEMS Immunol Med Microbiol. 2012;65:343-9.

13. Fusco A, Coretti L, Savio V, Buommino E, Lembo F, Donnarumma G. Biofilm formation and Immunomodulatory activity of Proteus mirabilis clinically isolated strains. Int J Mol Sci. 2017;18:414.

14. Donlan RM. Biofilms: microbial life on surfaces. Emerg Infect Dis. 2002;8:881.

15. Kwiecinska-Pirog J, Skowron K, Bartczak W, Gospodarek-Komkowska E. The ciprofloxacin impact on biofilm formation by Proteus mirabilis and $P$. vulgaris strains. Jundishapur J Microbiol. 2016;9:e32656. https://doi.org/10.5812/jjm. 32656.

16. Costerton JW, Stewart PS, Greenberg EP. Bacterial biofilms: a common cause of persistent infections. Science. 1999;284:1318-22.

17. McLean RJ, Lawrence JR, Korber DR, Caldwell DE. Proteus mirabilis biofilm protection against struvite crystal dissolution and its implications in struvite urolithiasis. J Urol. 1991;146:1138-42.

18. Rocha SP, Elias WP, Cianciarullo AM, Menezes MA, Nara JM, Piazza RM, et al. Aggregative adherence of uropathogenic Proteus mirabilis to cultured epithelial cells. FEMS Immunol Med Microbiol. 2007;51:319-26.

19. Stickler D, Ganderton L, King J, Nettleton J, Winters C. Proteus mirabilis biofilms and the encrustation of urethral catheters. Urol Res. 1993;21:407-11.

20. Jansen AM, Lockatell V, Johnson DE, Mobley HL. Mannose-resistant Proteuslike fimbriae are produced by most Proteus mirabilis strains infecting the urinary tract, dictate the in vivo localization of bacteria, and contribute to biofilm formation. Infect Immun. 2004;72:7294-305.

21. Ranjbar-Omid M, Arzanlou M, Amani M, Shokri Al-Hashem SK, Amir Mozafari $\mathrm{N}$, Peeri DH. Allicin from garlic inhibits the biofilm formation and urease activity of Proteus mirabilis in vitro. FEMS Microbiol Lett. 2015;362:fnv049

22. Rahal K, Wang F, Schindler J, Rowe B, Cookson B, Huovinen P, et al. Reports on surveillance of antimicrobial resistance in individual countries. Clin Infect Dis. 1997;24:S169-S75.

23. Alabi OS, Mendonça N, Adeleke OE, da Silva GJ. Molecular screening of antibiotic-resistant determinants among multidrug-resistant clinical isolates of Proteus mirabilis from SouthWest Nigeria. Afr Health Sci. 2017;17:356-65.

24. Bie $L$, Wu H, Wang $X H$, Wang $M, X u H$. Identification and characterization of new members of the SXT/R391 family of integrative and conjugative elements (ICEs) in Proteus mirabilis. Int J Antimicrob Agents. 2017;50:242-6.

25. Bameri Z, Karam MRA, Habibi M, Ehsani P, Bouzari S. Determination immunogenic property of truncated MrpH. FliC as a vaccine candidate against urinary tract infections caused by Proteus mirabilis. Microb Pathog. 2018;114:99-106

26. Jacobsen SM, Shirtliff ME. Proteus mirabilis biofilms and catheter-associated urinary tract infections. Virulence. 2011;2:460-5.

27. Qi L, Li H, Zhang C, Liang B, Li J, Wang L, et al. Relationship between antibiotic resistance, biofilm formation, and biofilm-specific resistance in Acinetobacter baumannii. Front Microbiol. 2016;7:483.

28. Kwiecinska-Piróg J, Bogiel T, Skowron K, Wieckowska E, Gospodarek E. Proteus mirabilis biofilm-qualitative and quantitative colorimetric methodsbased evaluation. Braz J Microbiol. 2014;45:1423-31.
29. Zhang W, Niu Z, Yin K, Liu P, Chen L. Quick identification and quantification of Proteus mirabilis by polymerase chain reaction (PCR) assays. Ann Microbiol. 2013;63:683-9.

30. Ali HH, Yousif MG. Detection of some virulence factors genes of Proteus mirablis that isolated from urinary tract infection. Int J. 2015;3:156-63.

31. Shi $X$, Lin $Y$, Qiu $Y, L i Y$, Jiang $M$, Chen $Q$, et al. Comparative screening of digestion tract toxic genes in Proteus mirabilis. PLoS One. 2016;11:e0151873.

32. Barbour EK, Hajj ZG, Hamadeh S, Shaib HA, Farran MT, Araj G, et al. Comparison of phenotypic and virulence genes characteristics in human and chicken isolates of Proteus mirabilis. Pathogens and global health. 2012; 106:352-7.

33. Zunino P, Geymonat L, Allen AG, Legnani-Fajardo C, Maskell DJ. Virulence of a Proteus mirabilis ATF isogenic mutant is not impaired in a mouse model of ascending urinary tract infection. FEMS Immunol Med Microbiol. 2000;29: $137-43$.

34. Zunino P, Sosa V, Allen AG, Preston A, Schlapp G, Maskell DJ. Proteus mirabilis fimbriae (PMF) are important for both bladder and kidney colonization in mice. Microbiology. 2003;149:3231-7.

35. Sosa V, Schlapp G, Zunino P. Proteus mirabilis isolates of different origins do not show correlation with virulence attributes and can colonize the urinary tract of mice. Microbiology. 2006;152:2149-57.

36. Stankowska D, Kwinkowski M, Kaca W. Quantification of Proteus mirabilis virulence factors and modulation by acylated homoserine lactones. J Microbiol Immunol Infect. 2008;41:243-53.

37. Wong MHY, Wan HY, Chen S. Characterization of multidrug-resistant Proteus mirabilis isolated from chicken carcasses. Foodborne Pathog Dis. 2013;10: $177-81$.

38. Harada K, Niina A, Shimizu T, Mukai Y, Kuwajima K, Miyamoto T, et al. Phenotypic and molecular characterization of antimicrobial resistance in Proteus mirabilis isolates from dogs. J Med Microbiol. 2014;63:1561-7.

39. Adamus-Bialek W, Zajac E, Parniewski P, Kaca W. Comparison of antibiotic resistance patterns in collections of Escherichia coli and Proteus mirabilis uropathogenic strains. Mol Biol Rep. 2013;40:3429-35.

40. Kanayama A, Kobayashi I, Shibuya K. Distribution and antimicrobial susceptibility profile of extended-spectrum $\beta$-lactamase-producing Proteus mirabilis strains recently isolated in Japan. Int J Antimicrob Agents. 2015;45: $113-8$.

41. Pedersen $K$, Pedersen $K$, Jensen $H$, Finster $K$, Jensen VF, Heuer OE. Occurrence of antimicrobial resistance in bacteria from diagnostic samples from dogs. J Antimicrob Chemother. 2007;60:775-81.

42. Huang $Y, X u Y$, Wang $Z$, Lin $X$. Antimicrobial resistance and genotype analysis of extended-Spectrum- $\beta$-lactamase-producing Proteus mirabilis. Open J Clin Diagnostics. 2014;4:57.

43. Okonko I, Nkang A, Fajobi E, Mejeha O, Udeze A, Motayo B, et al. Incidence of multi-drug resistant (MDR) organisms in some poultry feeds sold in Calabar Metropolis, Nigeria. Br J Pharmacol Toxicol. 2010;1:15-28.

44. Neupane S, Pant ND, Khatiwada S, Chaudhary R, Banjara MR. Correlation between biofilm formation and resistance toward different commonly used antibiotics along with extended spectrum beta lactamase production in uropathogenic Escherichia coli isolated from the patients suspected of urinary tract infections visiting Shree Birendra Hospital, Chhauni, Kathmandu, Nepal. Antimicrob Resist Infect Control. 2016;5:5.

45. Tajbakhsh E, Ahmadi P, Abedpour-Dehkordi E, Arbab-Soleimani N, Khamesipour F. Biofilm formation, antimicrobial susceptibility, serogroups and virulence genes of uropathogenic $E$. coli isolated from clinical samples in Iran. Antimicrob Resist Infect Control. 2016;5:11.

46. Shrestha LB, Bhattarai NR, Khanal B. Antibiotic resistance and biofilm formation among coagulase-negative staphylococci isolated from clinical samples at a tertiary care hospital of eastern Nepal. Antimicrob Resist Infect Control. 2017;6:89

47. Zhang J, Xu C, Shen H, Li J, Guo L, Cao G, et al. Biofilm formation in Haemophilus parasuis: relationship with antibiotic resistance, serotype and genetic typing. Res Vet Sci. 2014;97:171-5.

48. May $\mathrm{T}$, Ito A, Okabe $\mathrm{S}$. Induction of multidrug resistance mechanism in Escherichia coli biofilms by interplay between tetracycline and ampicillin resistance genes. Antimicrob Agents Chemother. 2009;53:4628-39.

49. Hall CW, Mah TF. Molecular mechanisms of biofilm-based antibiotic resistance and tolerance in pathogenic bacteria. FEMS Microbiol Rev. 2017; 41:276-301

50. Yue L. Identification of Proteus mirabilis from goat and detection of its major virulence genes Southwest University for nationalities; 2016. 
51. Gabidullin Z, Zhukova S, Ezepchuk I, Bondarenko V. The detection of a choleriform thermolabile enterotoxin in clinical strains of Proteus isolated in different infections. Zh Mikrobiol Epidemiol Immunobiol. 1989;12:14-6.

52. Songer J, Post K. Veterinary Microbiology: bacterial and fungal agents of animal disease. St Louis: Elsevier Saunders, St Louis; 2005. p. 687.

53. Leite A, Miguel M, Peixoto R, Ruas-Madiedo P, Paschoalin V, Mayo B, et al. Probiotic potential of selected lactic acid bacteria strains isolated from Brazilian kefir grains. J Dairy Sci. 2015;98:3622-32.

54. Peeters E, Nelis HJ, Coenye T. Comparison of multiple methods for quantification of microbial biofilms grown in microtiter plates. J Microbiol Methods. 2008;72:157-65.

55. Khoramian B, Jabalameli F, Niasari-Naslaji A, Taherikalani M, Emaneini M. Comparison of virulence factors and biofilm formation among Staphylococcus aureus strains isolated from human and bovine infections. Microb Pathog. 2015;88:73-7.

56. Bauer A, Kirby W, Sherris JC, Turck M. Antibiotic susceptibility testing by a standardized single disk method. Am J Clin Pathol. 1966:45:493.

57. Jean B. Patel, Franklin R. Cockerill III, et al. Performance Standards for Antimicrobial Susceptibility Testing; Twenty-Fifth Informational Supplement. CLSI Document M100-S25, Clinical and Laboratory Standards Institute. 2015; 35(3).

58. Magiorakos AP, Srinivasan A, Carey R, Carmeli Y, Falagas M, Giske C, et al. Multidrug-resistant, extensively drug-resistant and pandrug-resistant bacteria: an international expert proposal for interim standard definitions for acquired resistance. Clin Microbiol Infect. 2012;18:268-81.

59. Osman KM, Samir A, Abo-Shama UH, Mohamed EH, Orabi A, Zolnikov T. Determination of virulence and antibiotic resistance pattern of biofilm producing Listeria species isolated from retail raw milk. BMC Microbiol. 2016;16:263

60. Jing X, Gu S, Liu D, Zhao L, Xia S, He X, Chen H, Ge J. Lactobacillus brevis 23017 Relieves Mercury Toxicity in the Colon by Modulation of Oxidative Stress and Inflammation Through the Interplay of MAPK and NF-KB Signaling Cascades. Front Microbiol. 2018;9:2425.

\section{Publisher's Note}

Springer Nature remains neutral with regard to jurisdictional claims in published maps and institutional affiliations.

Ready to submit your research? Choose BMC and benefit from:

- fast, convenient online submission

- thorough peer review by experienced researchers in your field

- rapid publication on acceptance

- support for research data, including large and complex data types

- gold Open Access which fosters wider collaboration and increased citations

- maximum visibility for your research: over $100 \mathrm{M}$ website views per year

At $\mathrm{BMC}$, research is always in progress.

Learn more biomedcentral.com/submissions 